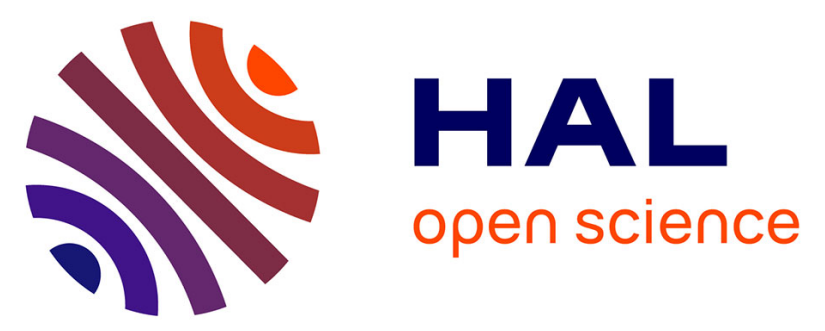

\title{
Quantitative evaluation of liver metastases density on computed tomography: A new tool to evaluate early response to bevacizumab-containing chemotherapy
}

Thibault Mazard, Eric Assénat, Marie Dupuy, Caroline Mollevi, Amandine

René, Antoine Adenis, Bruno Chauffert, Eveline Boucher, Eric Francois, Jean-Yves Pierga, et al.

\section{To cite this version:}

Thibault Mazard, Eric Assénat, Marie Dupuy, Caroline Mollevi, Amandine René, et al.. Quantitative evaluation of liver metastases density on computed tomography: A new tool to evaluate early response to bevacizumab-containing chemotherapy. Digestive and Liver Disease, 2019, 51 (8), pp.1185-1191. 10.1016/j.dld.2019.03.028 . hal-02452602

\section{HAL Id: hal-02452602 \\ https://hal.umontpellier.fr/hal-02452602}

Submitted on 25 Oct 2021

HAL is a multi-disciplinary open access archive for the deposit and dissemination of scientific research documents, whether they are published or not. The documents may come from teaching and research institutions in France or abroad, or from public or private research centers.
L'archive ouverte pluridisciplinaire HAL, est destinée au dépôt et à la diffusion de documents scientifiques de niveau recherche, publiés ou non, émanant des établissements d'enseignement et de recherche français ou étrangers, des laboratoires publics ou privés.

\section{(ㄷ)(1) $\$$}

Distributed under a Creative Commons Attribution - NonCommerciall 4.0 International 
Quantitative evaluation of liver metastases density on computed tomography: a new tool to evaluate early response to bevacizumab-containing chemotherapy

\section{Thibault Mazard ${ }^{\text {a-b }}$, Eric Assenat ${ }^{\text {a-c }}$, Marie Dupuy ${ }^{c}$, Caroline Mollevi ${ }^{\text {b-d }}$, Amandine René ${ }^{\mathrm{f}}$, Antoine Adenis ${ }^{\text {a-b }}$, Bruno Chauffert ${ }^{\text {h }}$, Eveline Boucher ${ }^{\text { }}$, Eric Francois ${ }^{\text {j, Jean-Yves Pierga }}{ }^{\text {, }}$, Michel Ducreux ${ }^{1}$, Marc Ychou ${ }^{\text {a-b }}$, Benoit Gallix ${ }^{\text {e. }}$}

${ }^{a}$ Medical Oncology Unit, Institut régional du Cancer de Montpellier (ICM) - Val d'Aurelle, 208 avenue des Apothicaires, 34298 Montpellier cedex 5, France;

${ }^{\mathrm{b}}$ IRCM, Inserm, Univ Montpellier, ICM, Montpellier, France;

${ }^{c}$ Medical Oncology Unit, Hôpital Saint-Eloi, CHU de Montpellier, 80 avenue Augustin Fliche, 34295 Montpellier cedex 5, France;

${ }^{\mathrm{d}}$ Department of Biometry, Institut régional du Cancer de Montpellier (ICM) - Val d'Aurelle, 208 avenue des Apothicaires, 34298 Montpellier cedex 5, France;

eDepartement of Radiology, Hôpital Saint-Eloi, CHU de Montpellier, 80 avenue Augustin Fliche, 34295 Montpellier cedex 5, France;

${ }^{\mathrm{f}}$ Intrasense SA, 1231 Avenue du Mondial 98, 34000 Montpellier, France;

${ }^{\text {h } M e d i c a l ~ O n c o l o g y ~ U n i t, ~ C e n t r e ~ H o s p i t a l i e r ~ U n i v e r s i t a i r e ~ d ' A m i e n s, ~ P l a c e ~ V i c t o r ~ P a u c h e t, ~} 80054$ Amiens Cedex, France;

i Department of Digestive Oncology, Centre Eugène Marquis, Avenue de la Bataille FlandresDunkerque, CS 44229, 35042 Rennes cedex, France;

j Pôle de Médecine, Centre Antoine-Lacassagne, 33 Avenue Valombrose, 06189 Nice cedex 2, France;

${ }^{\mathrm{k}}$ Department of Medical Oncology, Institut Curie, 26 rue d'Ulm, 75248 Paris cedex 05, France;

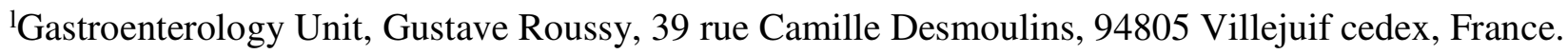


*Corresponding author: Dr Thibault Mazard

E-mail address: thibault.mazard@icm.unicancer.fr

Tel: +33 4-67-61-3029; Fax: +33 4-67-61-2347; Medical Oncology Unit, Institut régional du Cancer de Montpellier (ICM) - Val d'Aurelle, 208 avenue des Apothicaires, 34298 Montpellier cedex 5, France

Funding: This study was supported by a grant from Roche and CHU Montpellier-Nîmes, France.

Word count: 2895 words 


\begin{abstract}
Background: Response Evaluation Criteria In Solid Tumors (RECIST) are used to assess tumour shrinkage after cytotoxic chemotherapy, but may be inadequate for efficacy evaluation of antiangiogenic therapies.
\end{abstract}

Aims: This study aimed to identify novel radiologic tumour response criteria based on early changes in tumour size and density, observed on computed tomography (CT), in patients with colorectal liver metastases (CRLM) treated with bevacizumab-containing chemotherapy.

Methods: CT of 71 and 68 CRLM patients treated with bevacizumab and non-bevacizumab-based regimens, respectively, were retrospectively reviewed. Tumour size, tumour density, and tumour-toliver density (TTLD) ratio were determined at baseline and at first restaging. We tested their correlation with progression-free (PFS) and overall survival (OS) using the log-rank test.

Results: In the bevacizumab group, neither RECIST response nor tumour density variation was correlated with PFS or OS. In contrast, PFS and OS were significantly longer in patients with tumour size reduction $\geq 15 \%$ (RECIST-15\%) and/or decrease in TTLD ratio not exceeding -10\% (TTLD-10\%) than in patients who didn't reach any of those criteria, in univariate and multivariate analysis. Only size-response criteria predicted clinical outcome in the non-bevacizumab group. Conclusions: This study highlights new quantitative CT criteria that may early predict the efficacy of bevacizumab in CRLM patients.

Keywords: Bevacizumab; Contrast-enhanced computed tomography; Metastatic colorectal cancer; Tumour-to-liver density 


\section{Introduction}

Bevacizumab is a humanised monoclonal antibody targeting the vascular endothelial growth factor (VEGF). By inhibiting its function on vascular endothelial cells, it blocks the angiogenesis of solid tumours needed for tumour growth, activating invasion and metastasis. The addition of bevacizumab to fluoropyrimidine-based chemotherapy, with irinotecan or oxaliplatin, as first- and second-line treatment for metastatic colorectal cancer (mCRC) significantly increased the median progressionfree survival (PFS) in randomised controlled trials ${ }^{1-3}$.

However, the absence of predictive biomarkers prevents the early identification of patients who will most likely benefit from bevacizumab therapy. Moreover, standard Response Evaluation Criteria in Solid Tumors (RECIST) based on long-axis measurements on axial computed tomography (CT) may be inadequate while assessing the efficacy of bevacizumab in each individual patient ${ }^{4}$. Indeed, bevacizumab-based regimen can induce morphological tumour changes without significant modification in their sizes. They have been well described by Chun et al. ${ }^{5}$ in a cohort of patients with colorectal liver metastases (CRLM) before surgical resection and are characterised by a progressive transformation in lesions with homogeneous overall attenuation and sharp tumour-liver interface. In this study and a subsequent one ${ }^{6}$, they reported that patients with those optimal changes exhibited better pathologic responses and outcomes, whereas RECIST responders did not. However, concerns arise about the inter-site reproducibility and transferability of such qualitative and therefore subjective criteria.

Tumour density variation measurement may represent an objective mean to consider intratumoural modifications in response to anti-angiogenic therapies. In metastatic gastro-intestinal tumour (GIST), where RECIST criteria also significantly underestimated the response to imatinib, Choi et al. developed new CT evaluation criteria measuring CT attenuation coefficient that provide a good correlation with outcome ${ }^{7}$. 
Several studies also suggest the relevance of using alternative methods of categorising changes in size to early discriminate on-treatment good versus poor responders. Early tumour shrinkage (ETS), using $20 \%$ reduction in the sum of the longest diameters of target lesions as a cut-off on the first CT scan restaging, is a good predictor of long-term outcomes in mCRC patients treated with cytotoxic chemotherapy alone or combined with cetuximab ${ }^{8,9}$. Cremolini et al. ${ }^{10}$ showed similar correlations in patients treated with bevacizumab-based regimen.

In this context, this study aimed to further investigate the prognostic values of alternative CT-based quantitative assessment of early tumour response (size and density) in mCRC patients treated with a bevacizumab-containing regimen and in a similar population receiving regimens without antiangiogenic therapies.

\section{Materials and Methods}

\subsection{Study design}

We conducted a retrospective study reviewing the contrast-enhanced CT (CECT) images of a prospectively accrued cohort of patients with unresectable CRLM treated with a first-line therapy in three French multicentre clinical trials.

This work was performed in collaboration with the UNICANCER group and was approved by our institutional review board.

Written informed consent was obtained from each patient before joining the main study.

\subsection{Patient selection}

To be included in this study, patients had to have at least one liver metastasis larger than $1.5 \mathrm{~cm}$, a baseline $\mathrm{CT}$ evaluation and a restaging $\mathrm{CT}$ study of sufficient quality to allow scoring of density response. Images acquisition needs to be obtained after intravenous contrast injection during the 
portal venous phase with a slice thickness less than $3 \mathrm{~mm}$. The quality of contrast enhancement was assessed by analyzing attenuation of portal and hepatic veins. All CECT with a density of less than 100 Hounsfield Unit (HU) were excluded from analysis.

\section{Bevacizumab group}

Patients were selected from a randomised non-comparative Phase II trial (ACCORD 13 trial, NCT00423696) evaluating the efficacy and safety of bevacizumab in combination with XELIRI or FOLFIRI as first-line therapy ${ }^{11}$. Amongst the 145 patients enrolled in this trial, data from 71 patients were used in the present study. Reasons for exclusion from the analysis are detailed in Fig. 1.

\section{Non-bevacizumab group}

The non-bevacizumab group included 21 out of the 42 patients enrolled in the ERBIRINOX trial (NCT00556413) and 47 out of the 122 patients enrolled in the METHEP trial (NCT00208260) ${ }^{12,13 .}$ They were all treated according to different chemotherapy regimens without bevacizumab. Reasons for exclusion from the analysis are detailed in Fig. 1.

\subsection{Image analysis}

All the CT scans used in this study were anonymised. Liver target lesions were chosen on the pretreatment CT scan by a senior abdominal radiologist. He manually measured their long-axis diameters according to RECIST 1.1 criteria. Volumetric segmentations of the tumour were then performed using semi-automated edge detection software (Myrian®, Intrasense, Montpellier, France) by the same radiologist. Tumour edges were adjusted until satisfactory three-dimensional selection of a target lesion was obtained, and tumour mean volumetric attenuations were measured. The software also automatically segmented healthy liver, excluding the metastases and liver vessels, to calculate its 
mean density. To compensate the intra- and inter-individual heterogeneity in liver contrast enhancement, the tumour-to-liver density (TTLD) ratio was determined, that is, the mean tumour density divided by the mean healthy liver density. Same analyses were repeated on the first CT restaging, 2 weeks after the $\mathrm{CT}$ baseline review, and this was performed in a random order. The radiologist was not informed about the patients' clinical data and patients' baseline CT examination results.

\subsection{Statistical analysis}

Imaging parameters were dichotomised to ensure the best discrimination between patients with late (time until progression $>10$ months) and early disease progression (time until progression $\leq 10$ months). Using the receiving operator characteristic (ROC) curve methodology, optimal threshold values were calculated to maximise Youden's index.

Data were categorised according to frequency values for categorical variables and median and range values for continuous variables.

The primary endpoint for this study was PFS, calculated from the baseline CT scan date to disease progression or death from any cause. Patients who were alive without disease progression were censored at the date of last contact. Overall survival (OS) was the secondary endpoint and was calculated from same start point to death from any cause. Survival rates were estimated using the Kaplan-Meier method.

In univariate analysis, the log-rank test was used to identify the prognostic variables. Significant clinical and imaging parameters for PFS in a univariate analysis $(p<0.10)$ were included in a multivariate Cox proportional hazards model. A weight, proportional to the coefficient of the Cox model, was attributed for each significant variable and then added to obtain an overall score. The 
number of prognostic score modalities was reduced by regrouping non-significant adjacent categories. Finally, the obtained prognostic score was evaluated in the non-bevacizumab population.

\section{Results}

\subsection{Patient and responses}

\section{Bevacizumab group}

The analysed sample did not significantly differ from the whole clinical trial population (ACCORD 13 trial) in terms of age, sex, and PFS and OS (data not shown). Patient characteristics are detailed in Table 1.

Median time from pretreatment to first restaging CT scan was 2.1 months (range, 1-3.4 months). The pretreatment mean density of target lesions ranged from 32.4 to $107.02 \mathrm{HU}$ with a median of 64.05 HU. At first restaging, the mean tumor density ranged from 30.5 to $111.16 \mathrm{HU}$ with a median of 55.05 HU. The median mean healthy liver attenuation at baseline and at first restaging, was 105.69 HU and 98.45 HU, respectively (Supplementary table).

According to RECIST 1.1 criteria, $26.8 \%, 71.8 \%$, and $1.4 \%$ of patients presented with a partial response (PR), stable disease (SD), and progression disease (PD), respectively, at first restaging. The median tumour density was reduced by $11.3 \%$ (range, $-62.7-+39.8 \%$ ) and the TTLD ratio by $5.7 \%$ (range $-38.0-+43.4 \%$ ). With a median follow-up of 34.1 months (range, 2.8-47.5 months), median PFS and OS were 9.6 months (95\%CI: 9.1-10.1 months) and 22.1 months (95\%CI: 20.2-24.8 months), respectively.

\section{Non-bevacizumab group}

Median time from pretreatment to first restaging CT assessments was 2.0 months (range, 1.6-3.2 months). 
The pretreatment mean density of target lesions ranged from 43.2 to $117.3 \mathrm{HU}$ with a median of 76.73 HU. At first restaging, the mean tumor density ranged from 43.2 to $113.4 \mathrm{HU}$ with a median of 76.07 HU. The median mean healthy liver attenuation at baseline and at first restaging, was 111.63 HU and 106.79 HU, respectively (Supplementary table).

According to RECIST 1.1 criteria, $48.5 \%, 50.0 \%$, and $1.5 \%$ of the patients exhibited a PR, SD, and PD, respectively, at first restaging. The median tumour density was reduced by $1.1 \%$ (range $-37.7-$ $+48.3 \%$ ), and the TTLD ratio increased by $3.1 \%$ (range, $-40.9-+54.4 \%$ ). With a median follow-up of 45.4 months (range, 3.5-67.8 months), median PFS and OS were 11.8 months (95\% CI, 10.5-16.7 months) and 48.8 months (95\% CI, 27 months-not reached), respectively.

\subsection{Association between radiologic parameters and outcomes}

\section{Bevacizumab group}

Optimal ROC-determined cut-off points were -15\% for RECIST 1.1 measurements (RECIST-15\%), $0 \%$ for tumour variation density, and $-10 \%$ for TTLD (TTLD $-10 \%$ ) ratio.

On univariate analysis, measurements according to these RECIST $_{-15 \%}$ and TTLD $_{-10 \%}$ thresholds were significantly associated with both PFS and OS (Table 2). Size response measurements according to standard RECIST threshold (-30\%), ETS threshold (-20\%), and tumour variation density according to $0 \%$ threshold were not significantly correlated with survival.

The multivariate Cox regression confirmed that RECIST $_{-15 \%}$ and TTLD-10\% are strong independent prognostic factors. RECIST $_{-15 \%}$ responders (measurements < threshold) had significantly longer PFS (HR, 0.46; 95\% CI, 0.27-0.80; $p=0.007)$ and OS (HR, 0.46; 95\% CI, 0.26-0.82; $p=0.010)$ than RECIST $_{-15 \%}$ non-responders (measurements $\geq$ threshold). Similarly, PFS and OS in TTLD-10\% responders (measurements $\geq$ threshold) was significantly greater (HR, 0.44; 95\% CI, 0.26-0.76; 
$p=0.003$ and HR, $0.48 ; 95 \% \mathrm{CI}, 0.27-0.84 ; p=0.012$, respectively) as compared to TTLD-10\% nonresponders (measurements $<$ threshold) (Table 3).

\section{Non-bevacizumab group}

Patients with size response according to RECIST 1.1 criteria had longer PFS and OS. Size response according to a lower threshold (-15\% and $-20 \%)$ was only significantly correlated to OS. None of the tumour density variation criteria could predict outcomes in this group (Table 2 and Table 3).

\subsection{Development of a prognostic score for bevacizumab-treated patients}

The variables used to derive the prognostic scores were determined using multivariate analysis. We combined the two relevant factors, RECIST $_{-15 \%}$ and TTLD $_{-10 \%}$ thresholds, into a single prognostic index with values of 0 and 1 (Table 4). The index sums provided an overall score with values ranging from 0 to 2 . We subsequently defined two prognostic classes by grouping together populations of poor and intermediate prognosis $(\mathrm{PIP}=$ scores 1 and 2$)$ versus good prognosis $(\mathrm{GP}=$ score 0$)$. In the bevacizumab group, patients classified in the GP group had a significantly better PFS (10.3 months; 95\% CI, 9.4-11.3 months) and OS (30.7 months; 95\% CI, 21.9 months-not reached) than those classified in the PIP group (9.1 months; 95\% CI, 8.0-9.8 months and 20.4 months; $95 \%$ CI, 14.9-22.2 months for PFS and OS, respectively) (Table 4).

In the non-bevacizumab group, the prognostic score was neither correlated with PFS $(p=0.89)$ nor with OS ( $p=0.27)$. Kaplan-Meier survival curves by prognostic category are shown in Fig. 2.

\section{Discussion}

We report new radiologic response criteria that combine measurements of tumour size and density ratio variations on bevacizumab-containing chemotherapy in CRLM patients. Using ROC- 
determined cut-off points, we established $15 \%$ decrease in the maximum diameters of target lesions according to RECIST 1.1 criteria (RECIST-15\%) and 10\% decrease in TTLD ratio (TTLD-10\%) at the first restaging CT scan as optimal thresholds to predict long-term outcomes. Indeed, patients with a tumour size reduction superior or equal to $15 \%$ and a decrease in TTLD ratio not exceeding -10\% had longer PFS and OS compared to the ones who had no tumour reduction. Moreover, that correlation was only noticed in the bevacizumab group, suggesting a better ability of those criteria to identify bevacizumab-specific anti-tumour effect.

This study illustrates again an inadequacy of conventional RECIST-defined response to early identify anti-angiogenic-based treatment responders. That can be partly corrected by the use of a smaller cutoff value size reduction than the traditional $30 \%$. Before this study, several studies have shown that reaching a tumour shrinkage greater than or equal to $\geq 15-20 \%$ at first restaging was correlated with improved PFS and OS in first-line anti-angiogenic plus chemotherapy-treated patients ${ }^{10,14,15}$.

In order to circumvent size measurement shortcomings, CECT can also provide other non-invasive quantitative parameters to more specifically characterise and quantify the effect of bevacizumab on tumour angiogenesis. Indeed, effective inhibition of that process must logically lead to changes in tumour perfusion, blood volume, and capillary permeability, resulting in post-contrast attenuation (or density) changes that can be sequentially measured on CT follow-up ${ }^{16}$. Additionally, reduction in tumour density has already been shown to be an additional indicator of early response to antiangiogenic therapy in a variety of non-colorectal tumours. The first illustration was obtained by Choi et al. in patients with GIST treated with imatinib, where a decrease greater than or equal to $15 \%$ of mean tumour density on first restaging of CECT could identify patients with more favourable outcomes ${ }^{7}$. An early decrease in tumour attenuation, alone or in combination with size and/or morphologic criteria, was also found to be correlated with survival outcomes in patients with metastatic renal cell carcinoma treated with anti-angiogenic targeted therapy ${ }^{17}$. In colorectal cancer, 
a retrospective study carried out by Chung et al. demonstrated that in 59 patients with liver metastases, evaluating treatment response at 2 months, with tumour size and density changes on CT, was a better predictor of time to tumour progression than changes in tumour size or density alone in two populations treated or not with bevacizumab ${ }^{18}$. In our study, reduction in tumour density was more pronounced in the bevacizumab group than in the non-bevacizumab group. Nevertheless, regardless of the applied threshold, we could not correlate that reduction with survival outcomes because absolute tumour density may have been influenced by variations in scanning parameters such as time of slice acquisition, dose and flow rate of injected iodinated contrast media from one CT examination to another one. As a result, we decided to normalise tumour density by the surrounding liver tissue density and called it TTLD assessment. Surprisingly, bevacizumab improved the survival outcomes in patients whose on-treatment ratio at 2 months did not decrease more than $10 \%$, that is, in patients with the maintenance of a certain degree of tumour enhancement and hence a certain degree of tumour blood supply compared to the healthy liver. This finding supports the vasculature 'normalization' hypothesis developed by Jain et al. ${ }^{19}$ to explain the anti-tumour effect of antivascular agents. Indeed, he argues that rather than inducing tumour vessel regression to starve the tumour, they were effective by correcting tumour vascular abnormalities thereby alleviating tumour hypoxia, facilitating tumour delivery of associated cytotoxic drugs, or reprogramming the immunosuppressive tumour micro-environment to an immunosupportive one.

Through this ratio, we also investigate the potential impact of surrounding healthy liver modifications under anti-angiogenic treatment on the whole treatment anti-tumour effect. In patients treated for liver metastases from neuroendocrine tumours, bevacizumab did not modify the perfusion parameters in normal liver tissue ${ }^{20}$. However, it was also found that bevacizumab attenuated hepatic fibrosis in rats ${ }^{21}$ and could protect against the occurrence of sinusoidal obstruction syndrome ${ }^{22}$, thereby perhaps facilitating tumour drug access. 
Over the past few years, other alternative radiologic criteria have been studied to address issues encountered with the efficacy assessment of anti-angiogenic therapies. We recently confirmed that MD Anderson morphologic criteria were able to early predict PFS in patients with unresectable CRLM treated with bevacizumab-containing chemotherapy and offered to add their evaluation to the established RECIST criteria ${ }^{23}$. Functional imaging has also the potential to give a greater insight of early changes of the vascular network in response to anti-VEGF pathway therapies. Contrastenhanced liver ultrasound has been the most studied modality in metastatic colorectal patients receiving first-line bevacizumab-based treatment. Several haemodynamic parameters were found to correlate with response and/or survival rates, but, most of the time, only in a small sample of patients and with the lack of control group without anti-angiogenic therapies ${ }^{24-26}$.

Furthermore, a major obstacle to the widespread use of those techniques is their reproducibility. Our method, although dependent on imaging quality, has the advantages of being easily and quickly applied, even by a non-radiologist physician, and the software used ensures continued accuracy and reduces inter-observer variability. Those different methods should actually be seen as complementary rather than competing, and a joint cross-validation study should be performed to optimise both approaches.

Even though data were prospectively collected, limitations of our study were as follows: its retrospective nature and the heterogeneity of the non-bevacizumab control group. The quality of CT images was also not homogeneous amongst the study cohorts, and because of the low quality of some CT scan images, we had to exclude many CT scans from the analysis. Another limitation is the lack of data exploring the correlation between our radiologic response criteria and pathological response that has consistently been found as a strong predictor of survival in patients with CRLM that was resected after chemotherapy ${ }^{27-29}$. Moreover, the coarse assessment performed by the software is not able to capture some pathological features described as predictive of patient outcomes in a context of 
bevacizumab-based treatment as the residual tumour thickness at the interface between the tumour and the non-neoplastic liver or the growth patterns (replacement/pushing/desmoplastic histopathologic growth pattern) also defined from pathological characteristics noticed at this interface 30,31 .

In conclusion, we report new quantitative radiologic parameters able to early identify patients with a more favourable outcome in a liver metastatic colorectal population treated with bevacizumab. Further validation in other cohorts is underway. 


\section{Acknowledgments}

The study was partly funded by a grant from Roche laboratory and Montpellier Universitary Hospital.

\section{References}

1. Hurwitz H, Fehrenbacher L, Novotny $\mathrm{W}$ et al. Bevacizumab plus irinotecan, fluorouracil, and leucovorin for metastatic colorectal cancer. N Engl J Med 2004;2335-42.

2. Saltz LB, Clarke S, Diaz-Rubio E et al. Bevacizumab in combination with oxaliplatin-based chemotherapy as first-line therapy in metastatic colorectal cancer: a randomized phase III study. J Clin Oncol 2008;2013-9.

3. Bennouna J, Sastre J, Arnold D, et al. Continuation of bevacizumab after first progression in metastatic colorectal cancer (ML18147): a randomised phase 3 trial. Lancet Oncol 2013;14(1):29-37.

4. Grothey A, Hedrick EE, Mass RD, et al. Response-independent survival benefit in metastatic colorectal cancer: a comparative analysis of N9741 and AVF2107. J Clin Oncol 2008;26(2):183-9.

5. Chun YS, Vauthey J-N, Boonsirikamchai P, et al. Association of computed tomography morphologic criteria with pathologic response and survival in patients treated with bevacizumab for colorectal liver metastases. JAMA 2009;302(21):2338-44.

6. Shindoh J, Loyer EM, Kopetz S, et al. Optimal morphologic response to preoperative chemotherapy: an alternate outcome end point before resection of hepatic colorectal metastases. J Clin Oncol 2012;30(36):4566-72.

7. Choi H, Charnsangavej C, Faria SC, et al. Correlation of computed tomography and positron emission tomography in patients with metastatic gastrointestinal stromal tumor treated at a single institution with imatinib mesylate: proposal of new computed tomography response criteria. J Clin Oncol 2007;25(13):1753-9.

8. Giessen C, Laubender RP, Fischer von Weikersthal L, et al. Early tumor shrinkage in metastatic colorectal cancer: Retrospective analysis from an irinotecan-based randomized first-line trial. Cancer Sci 2013;104(6):718-724.

9. Piessevaux H, Buyse M, Schlichting M, et al. Use of early tumor shrinkage to predict long-term outcome in metastatic colorectal cancer treated with cetuximab. J Clin Oncol 2013;31(30):3764-3775. 
10. Cremolini C, Loupakis F, Antoniotti C, et al. Early tumor shrinkage and depth of response predict long-term outcome in metastatic colorectal cancer patients treated with first-line chemotherapy plus bevacizumab: results from phase III TRIBE trial by the Gruppo Oncologico del Nord Ovest. Ann Oncol 2015;26(6):1188-94.

11. Ducreux M, Adenis A, Pignon J-P, et al. Efficacy and safety of bevacizumab-based combination regimens in patients with previously untreated metastatic colorectal cancer: Final results from a randomised phase ii study of bevacizumab plus 5-fluorouracil, leucovorin plus irinotecan versus bevacizumab plus capecitabine plus irinotecan (FNCLCC ACCORD 13/0503 study). Eur J Cancer 2013;49(6):1236-45.

12. Assenat E, Desseigne F, Thezenas S, et al. Cetuximab plus FOLFIRINOX (ERBIRINOX) as first-line treatment for unresectable metastatic colorectal cancer: a phase II trial. The Oncologist 2011;16(11):1557-64.

13. Ychou M, Rivoire $\mathrm{M}$, Thezenas $\mathrm{S}$, et al. A randomized phase II trial of three intensified chemotherapy regimens in first-line treatment of colorectal cancer patients with initially unresectable or not optimally resectable liver metastases. The METHEP trial. Ann Surg Oncol 2013;

14. Saad ED, Coart E, Sommeijer DW, et al. Early predictors of improved long-term outcomes in first-line antiangiogenics plus chemotherapy (anti-ANG/CT) in metastatic colorectal cancer (mCRC): Analysis of individual patient (pt) data from the ARCAD database. ASCO Meet Abstr 2014;32(15_suppl):3578.

15. Stintzing S, Modest DP, Rossius L, et al. FOLFIRI plus cetuximab versus FOLFIRI plus bevacizumab for metastatic colorectal cancer (FIRE-3): a post-hoc analysis of tumour dynamics in the final RAS wild-type subgroup of this randomised open-label phase 3 trial. Lancet Oncol 2016;17(10):1426-34.

16. Miles KA. Tumour angiogenesis and its relation to contrast enhancement on computed tomography: a review. Eur J Radiol 1999;30(3):198-205.

17. Bex A, Fournier L, Lassau N, et al. Assessing the response to targeted therapies in renal cell carcinoma: technical insights and practical considerations. Eur Urol 2014;65(4):766-77.

18. Chung W-S, Park M-S, Shin SJ, et al. Response evaluation in patients with colorectal liver metastases: RECIST version 1.1 versus modified CT criteria. Am J Roentgenol 2012;199(4):809-15.

19. Jain RK. Normalizing tumor vasculature with anti-angiogenic therapy: A new paradigm for combination therapy. Nat Med 2001;7(9):987-9.

20. Ng CS, Wei W, Duran C, et al. CT perfusion in normal liver and liver metastases from neuroendocrine tumors treated with targeted antivascular agents. Abdom Radiol 2018;43(7):1661-9. 
21. Huang Y, Feng H, Kan T, et al. Bevacizumab attenuates hepatic fibrosis in rats by inhibiting activation of hepatic stellate cells. PLoS ONE 2013;8(8):e73492.

22. Hubert C, Sempoux C, Humblet Y, et al. Sinusoidal obstruction syndrome (SOS) related to chemotherapy for colorectal liver metastases: factors predictive of severe SOS lesions and protective effect of bevacizumab. HPB 2013;15(11):858-64.

23. Mazard T, Boonsirikamchai P, Overman MJ, et al. Comparison of early radiological predictors of outcome in patients with colorectal cancer with unresectable hepatic metastases treated with bevacizumab. Gut 2018;67(6):1095-102.

24. Tranquart F, Dujardin P-A, Bouché O, et al. Value of contrast-enhanced ultrasound quantification criteria for identifying patients not responding to bevacizumab-based therapy for colorectal liver metastases.Ultraschall Med 2018 Oct;39(5):544-558.

25. Wu Z, Yang X, Chen L, et al. Anti-angiogenic therapy with contrast-enhanced ultrasound in colorectal cancer patients with liver metastasis: Medicine (Baltimore) 2017;96(20):e6731.

26. Amadori M, Barone D, Scarpi E, et al. Dynamic contrast-enhanced ultrasonography (D-CEUS) for the early prediction of bevacizumab efficacy in patients with metastatic colorectal cancer. Eur Radiol 2018;28(7):2969-78.

27. Rubbia-Brandt L, Giostra E, Brezault C, et al. Importance of histological tumor response assessment in predicting the outcome in patients with colorectal liver metastases treated with neo-adjuvant chemotherapy followed by liver surgery. Ann Oncol 2007;18(2):299-304.

28. Blazer DG 3rd, Kishi Y, Maru DM, et al. Pathologic response to preoperative chemotherapy: a new outcome end point after resection of hepatic colorectal metastases. J Clin Oncol 2008;26(33):5344-51.

29. Gruenberger T, Arnold D, Rubbia-Brandt L et al. Pathologic response to bevacizumabcontaining chemotherapy in patients with colorectal liver metastases and its correlation with survival. Surg Oncol 2012;21(4):309-15.

30. Maru DM, Kopetz S, Boonsirikamchai P, et al. Tumor thickness at the tumor-normal interface: a novel pathologic indicator of chemotherapy response in hepatic colorectal metastases. Am $\mathbf{J}$ Surg Pathol 2010;34(9):1287-1294.

31. Frentzas S, Simoneau E, Bridgeman VL, et al. Vessel co-option mediates resistance to antiangiogenic therapy in liver metastases. Nat Med 2016;22(11):1294-302. 


\section{Figure legends}

Figure 1. Study flowchart

ACCORD 13 Actions Concertées dans les Cancers COloRectaux et Digestifs n ${ }^{\circ} 13$ - Concerted Actions in COlorectal and Digestive Cancers $\mathrm{n}^{\circ} 13$ (NCT00423696), CT computed tomography, ERBIRINOX ERBitux IRINotecan OXaliplatin (NCT00556413), METHEP METastases HEPatic (NCT00208260).

Figure 2. Kaplan-Meier survival curves according to the prognostic score category in the bevacizumab $(\mathrm{A}+\mathrm{B})$ and the non-bevacizumab $(\mathrm{C}+\mathrm{D})$ groups

Prognostic scores according to RECIST (-15\% threshold) and tumour-to-liver density (-10\% threshold) ratio variations at first restaging compared to baseline measurement: score $0=$ good prognosis, score $1=$ intermediate prognosis, score $2=$ poor prognosis

Abbreviations: $O S$ overall survival, $P F S$ progression-free survival 


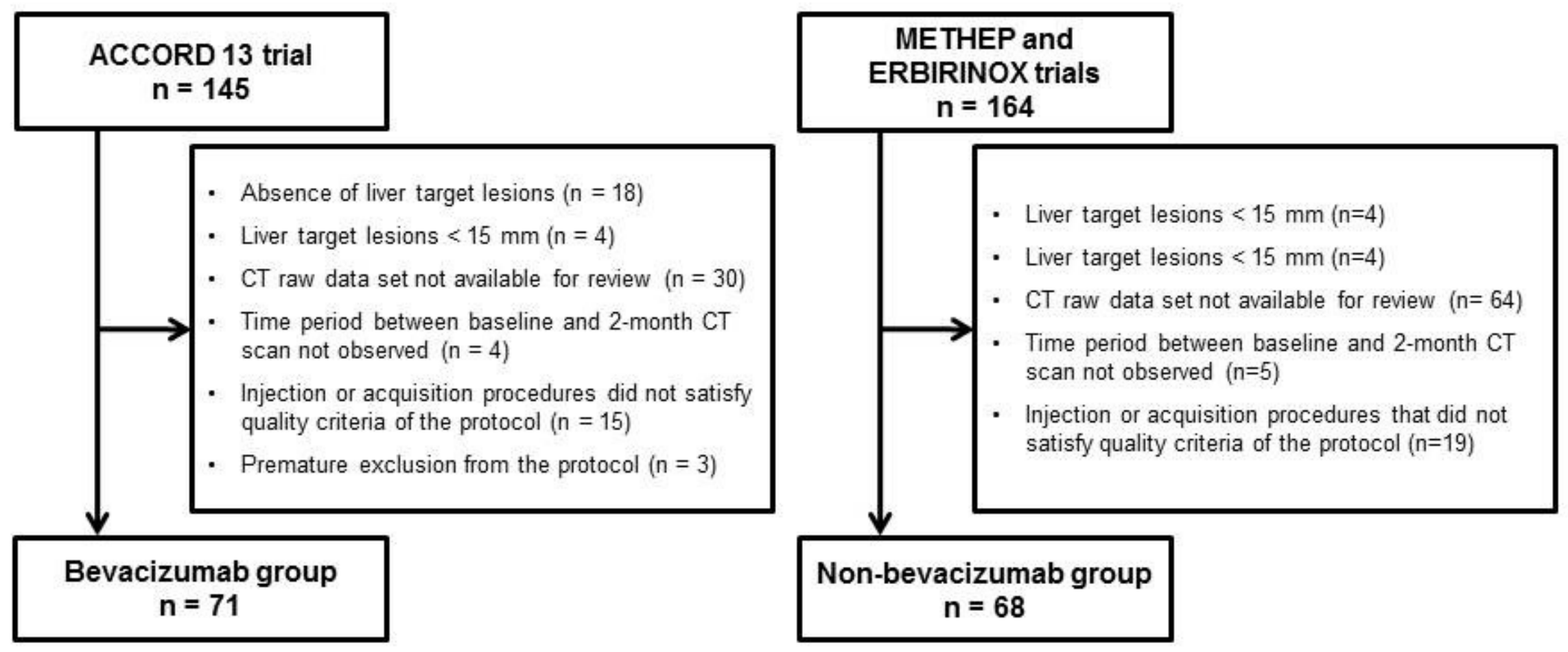




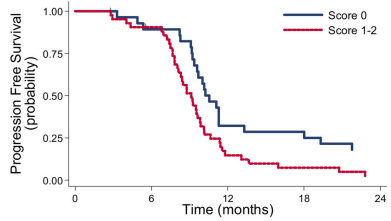

No. at risk Score 028 Score 1-2 43

C: PFS in the non-bevacizumab group

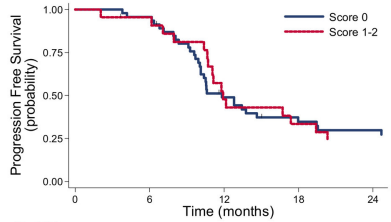

No. at risk Score $0 \quad 46$ Score 1-2 22

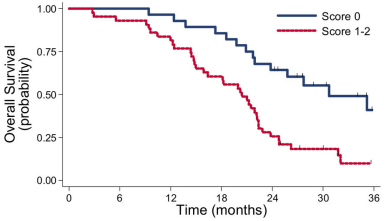

No. at risk

Score 028 Score 1-2 43

D: OS in the non-bevacizumab group

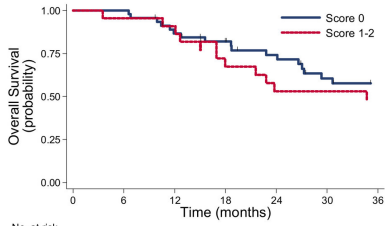

No. at risk

Score 046

Score 1-2 22 


\section{Table 1}

Patient demographics and tumour characteristics

\begin{tabular}{|c|c|c|}
\hline & $\begin{array}{c}\text { BEV group } \\
(N=71)\end{array}$ & $\begin{array}{c}\text { Non-BEV group } \\
\quad(N=68)\end{array}$ \\
\hline & $N(\%)$ & $N(\%)$ \\
\hline Age (years), median [range] & $62.0[39-75]$ & $60.5[32-81]$ \\
\hline$<60$ & $28(39)$ & $31(45)$ \\
\hline [60-69] & $27(38)$ & $29(43)$ \\
\hline [70-81] & $16(23)$ & $8 \quad(12)$ \\
\hline Males & $34(48)$ & $30(44)$ \\
\hline \multicolumn{3}{|l|}{ ECOG performance status } \\
\hline 0 & $38(54)$ & 49 (73) \\
\hline 1 & $28(39)$ & $18(27)$ \\
\hline 2 & $5 \quad(7)$ & -- \\
\hline Missing & & 1 \\
\hline \multicolumn{3}{|l|}{ Number of metastatic sites } \\
\hline 1 & $30(42)$ & $49(72)$ \\
\hline 2 & $33(47)$ & $11(16)$ \\
\hline$\geq 3$ & $8 \quad(11)$ & $8 \quad(12)$ \\
\hline \multicolumn{3}{|l|}{$\begin{array}{l}\text { Localisation of the primary } \\
\text { tumour }\end{array}$} \\
\hline Right side & $22(31)$ & $21(31)$ \\
\hline Left side & $45(63)$ & $44(65)$ \\
\hline Transverse & $4(6)$ & $3(4)$ \\
\hline \multicolumn{3}{|l|}{ Chemotherapy regimen } \\
\hline FOLFIRI with bevacizumab & $44(62)$ & -- \\
\hline XELIRI + bevacizumab & $27(38)$ & -- \\
\hline FOLFIRI standard & -- & $5(7)$ \\
\hline FOLFIRI high dose & -- & $13(19)$ \\
\hline FOLFOX 4 & -- & $5(7)$ \\
\hline FOLFOX 7 & -- & 7 (11) \\
\hline FOLFIRINOX & -- & $17(25)$ \\
\hline FOLFIRINOX with cetuximab & -- & $21(31)$ \\
\hline
\end{tabular}

Abbreviations: BEV bevacizumab, ECOG Eastern Cooperative Oncology Group, FOLFIRI folinic acid + 5-fluorouracile + irinotecan, FOLFIRINOX folinic acid + 5-fluorouracile + irinotecan + oxaliplatin, FOLFOX folinic acid + 5-fluorouracile + oxaliplatin, XELIRI capecitabine + irinotecan 
Table 2

Univariate analysis for PFS and OS

\begin{tabular}{|c|c|c|c|}
\hline \multicolumn{2}{|c|}{ BEV group $(N=71)$} & \multicolumn{2}{|c|}{ Non-BEV group $(N=68)$} \\
\hline PFS & OS & PFS & OS \\
\hline \multicolumn{2}{|c|}{ No. of events } & \multicolumn{2}{|c|}{ No. of events } \\
\hline
\end{tabular}

\begin{tabular}{|c|c|c|c|c|}
\hline \multicolumn{5}{|l|}{ Age, years } \\
\hline$<60$ & $26 / 28$ & $19 / 28$ & $24 / 31$ & $15 / 31$ \\
\hline [60-69] & $25 / 27$ & $19 / 27$ & $21 / 29$ & $14 / 29$ \\
\hline [70-81] & $15 / 16$ & $13 / 16$ & $4 / 8$ & $4 / 8$ \\
\hline p value & .056 & .086 & .464 & .954 \\
\hline \multicolumn{5}{|c|}{ ECOG performance status } \\
\hline 0 & $34 / 38$ & $25 / 38$ & $35 / 49$ & $24 / 49$ \\
\hline 1 & $27 / 28$ & $21 / 28$ & $14 / 18$ & $9 / 18$ \\
\hline 2 & $5 / 5$ & $5 / 5$ & - & - \\
\hline p value & .075 & .414 & .822 & .661 \\
\hline \multicolumn{5}{|c|}{ RECIST $_{-30 \%}$} \\
\hline$<-30 \%$ & $18 / 19$ & $11 / 19$ & $19 / 33$ & $12 / 33$ \\
\hline$\geq-30 \%$ & $48 / 52$ & $40 / 52$ & $30 / 35$ & $21 / 35$ \\
\hline p value & .949 & .262 & $.032 *$ & $.020^{*}$ \\
\hline \multicolumn{5}{|c|}{ RECIST $_{-15 \%}$} \\
\hline$<-15 \%$ & $44 / 47$ & $30 / 47$ & $38 / 56$ & $24 / 56$ \\
\hline$\geq-15 \%$ & $22 / 24$ & $21 / 24$ & $11 / 12$ & $9 / 12$ \\
\hline p value & $.028 *$ & $.009 *$ & .120 & $.040^{*}$ \\
\hline \multicolumn{5}{|c|}{ RECIST $_{-20 \%}$} \\
\hline$<-20 \%$ & $36 / 38$ & $25 / 38$ & $36 / 53$ & $22 / 53$ \\
\hline$\geq-20 \%$ & $30 / 33$ & $26 / 33$ & $13 / 15$ & $11 / 15$ \\
\hline p value & .727 & .265 & .262 & $.030^{*}$ \\
\hline \multicolumn{5}{|c|}{ Tumour density variation $_{0 \%}$} \\
\hline$<0 \%$ & $56 / 57$ & $43 / 57$ & $24 / 36$ & $18 / 36$ \\
\hline$\geq 0 \%$ & $10 / 14$ & $8 / 14$ & $26 / 32$ & $15 / 32$ \\
\hline p value & .095 & .289 & .357 & .880 \\
\hline \multicolumn{5}{|c|}{ Tumour density variation. $10 \%$} \\
\hline$<-10 \%$ & $39 / 40$ & $32 / 40$ & $14 / 20$ & $11 / 20$ \\
\hline$\geq-10 \%$ & $27 / 31$ & $19 / 31$ & $35 / 48$ & $22 / 48$ \\
\hline$p$ value & .099 & .563 & .588 & .635 \\
\hline \multicolumn{5}{|c|}{ Tumour density variation. $15 \%$} \\
\hline$<-15 \%$ & $26 / 27$ & $24 / 27$ & $4 / 13$ & $7 / 13$ \\
\hline$\geq-15 \%$ & $40 / 44$ & $27 / 44$ & $41 / 55$ & $26 / 55$ \\
\hline p value & .196 & .163 & .359 & .802 \\
\hline \multicolumn{5}{|l|}{ TTLD-10\% $_{-10}$} \\
\hline$<-10 \%$ & $27 / 28$ & $24 / 28$ & $8 / 12$ & $6 / 12$ \\
\hline$\geq-10 \%$ & $39 / 43$ & $27 / 43$ & $41 / 56$ & $27 / 56$ \\
\hline p value & $.013 *$ & $.012 *$ & .330 & .977 \\
\hline
\end{tabular}

Abbreviations: $B E V$ bevacizumab, $C R$ complete response, ECOG Eastern Cooperative Oncology Group, $P F S$ progression-free survival, $O S$ overall survival, $P D$ progressive disease, $P R$ partial response, $T T L D$ tumour-to-liver density

$* p \leq 0.05$ 
Table 3

Multivariate Cox regression analysis of relevant parameters (hazard ratios)

BEV group $(N=71)$

PFS

\section{RECIST-15\%}

$\geq-15 \%$

2.15

(1.25-3.70)

$0.007 *$

TTLD-10\%

$$
\begin{aligned}
& \geq-10 \% \\
& <-10 \%
\end{aligned}
$$$$
2.25
$$$$
(1.32-3.84)
$$

p value
(1.40-5.09)

$0.010^{*}$

OS

1
-
2.67
$40-5.09)$
$0.010^{*}$

\section{1}

4.14

(1.69-10.10)

$0.012 *$

\section{Non-BEV group $(N=68)$}

PFS

OS

$\begin{array}{cc}1 & 1 \\ - & - \\ 1.69 & 2.22 \\ (0.86-3.34) & (1.02-4.87) \\ 0.325 & 0.915\end{array}$

1

1.05

$(0.32-1.48) \quad(0.43-2.55)$

$0.148 \quad 0.059$

Data in parentheses are $95 \%$ confidence intervals. Abbreviations: $B E V$ bevacizumab, $C I$ confidence interval, PFS progression-free survival, OS overall survival, TTLD tumour-to-liver density

$*$ p value $\leq 0.05$ 
Table 4

Prognostic score

\begin{tabular}{|c|c|c|}
\hline \multirow[b]{2}{*}{ Score points } & \multicolumn{2}{|c|}{ Variable } \\
\hline & RECIST $_{-15 \%}$ & $\begin{array}{c}\text { TTLD. } \\
10 \% \\
\end{array}$ \\
\hline \multirow[t]{2}{*}{$\mathbf{0} \Rightarrow$ Good prognosis } & $<-15 \%$ & $\geq-10 \%$ \\
\hline & $<-15 \%$ & $<-10 \%$ \\
\hline \multirow{2}{*}{$1 \Rightarrow$ Intermediate prognosis } & \multicolumn{2}{|l|}{ or } \\
\hline & $\geq-15 \%$ & $\geq-10 \%$ \\
\hline $2 \Rightarrow$ Poor prognosis & $\geq-15 \%$ & $<-10 \%$ \\
\hline
\end{tabular}

Survival according to the prognostic category

\begin{tabular}{|c|c|c|c|c|c|c|c|c|c|c|}
\hline & \multicolumn{5}{|c|}{ BEV group $(N=71)$} & \multicolumn{5}{|c|}{ Non-BEV group $(N=68)$} \\
\hline & \multirow[b]{2}{*}{$\begin{array}{l}N \\
(\%) \\
\end{array}$} & \multicolumn{2}{|c|}{ PFS } & \multicolumn{2}{|c|}{ OS } & \multirow[b]{2}{*}{$\begin{array}{c}N \\
(\%)\end{array}$} & \multicolumn{2}{|c|}{ PFS } & \multicolumn{2}{|c|}{ OS } \\
\hline & & $\begin{array}{l}\text { Median } \\
(\mathrm{mo})\end{array}$ & HR & $\begin{array}{c}\text { Median } \\
(\text { mo })\end{array}$ & HR & & $\begin{array}{l}\text { Median } \\
(\mathrm{mo})\end{array}$ & HR & $\begin{array}{l}\text { Median } \\
(\mathrm{mo})\end{array}$ & HR \\
\hline $\begin{array}{l}\text { Intermediate }(1)+ \\
\text { poor prognosis }(2)\end{array}$ & $\begin{array}{c}43 \\
(60.6)\end{array}$ & 9.1 & 1 & 20.4 & 1 & $\begin{array}{c}22 \\
(32.3)\end{array}$ & 11.9 & 1 & 34.7 & 1 \\
\hline $\begin{array}{l}\text { Good prognosis } \\
\text { (0) }\end{array}$ & $\begin{array}{c}28 \\
(39.4)\end{array}$ & 10.3 & $\begin{array}{c}0.52 \\
(0.31- \\
0.87)\end{array}$ & 30.7 & $\begin{array}{c}0.35 \\
(0.19- \\
0.65)\end{array}$ & $\begin{array}{c}46 \\
(67.7)\end{array}$ & 11.8 & $\begin{array}{c}0.96 \\
(0.53- \\
1.73)\end{array}$ & 48.9 & $\begin{array}{c}0.67 \\
(0.33- \\
1.36)\end{array}$ \\
\hline
\end{tabular}

Data in parentheses are $95 \%$ confidence intervals unless otherwise stated. Abbreviations: $B E V$ bevacizumab, $C I$ confidence interval, $H R$ hazard ratio, mo months, $P F S$ progression-free survival, $O S$ overall survival, $T T L D$ tumour-to-liver density 